\title{
VARIATIONAL PRINCIPLES IN THE LINEAR DYNAMIC THEORY OF VISCOELASTICITY*
}

\author{
BY \\ MARSHALL J. LEITMAN \\ Brown University
}

1. Introduction. In a recent paper Gurtin [1] deduces variational principles which characterize the standard initial-value problems of linear elastodynamics.

Here we extend ${ }^{* *}$ these principles to dynamic viscoelasticity theory.

Notational agreements and mathematical preliminaries are given in Section 2. In Section 3 we formulate the initial-history problem for the dynamic linear theory of viscoelasticity and deduce two equivalent characterizations of its solutions. These alternate formulations, aside from being essential to the results presented here, are of interest in themselves. In Sections 4 and 5 we prove three variational principles applicable to the mixed boundary-value problem. The first of these is quite general and the admissible states are required to satisfy only the initial history condition. In the second, the admissible states, in addition, must satisfy the strain-displacement relations. The third variational principle is concerned only with the stresses.

We allow the state history to be of infinite duration. Those cases for which the history may be considered finite fall as a special case of the results presented. We also remark that for histories of finite duration variational principles may be deduced which have a simpler form. They will, however, require separate proof.

We do not, in this paper, give all the counterparts of the results in [1], since it is clear from those presented how the others may be obtained.

2. Notation and mathematical preliminaries. We will try to use, whenever possible, the notation developed in [1].

Indicial notation is used throughout. Thus, subscripts have the range of the integers 1, 2, 3 and denote the Cartesian components of vector- and tensor-valued functions; summation over repeated subscripts is implied and subscripts preceded by a comma indicate differentiation with respect to the corresponding Cartesian coordinate. Parentheses about a pair of free subscripts denote symmetry with respect to these subscripts, e.g. $u_{(i, i)}=\frac{1}{2}\left(u_{i, i}+u_{i, i}\right)$.

$R$ is an open bounded region in three-dimensional Euclidean space with the closure $R^{c}$ and boundary $\partial R . B_{u}$ and $B_{s}$ denote disjoint sets whose union is $\partial R$ and $n_{i}$ is the unit outward normal to $\partial R$. We write $x_{i}$ for a point of $R^{c}$ and use the abbreviation $x=\left(x_{1}, x_{2}, x_{3}\right)$.

A superscript indicates differentiation with respect to the time $t$.

Let $f$ and $g$ be scalar-valued functions defined on $R^{c} \times(-\infty, \infty)$. Then the convolution $f * g$ is the function on $R^{c} \times[0, \infty)$ defined by

$$
f * g(x, t)=\int_{0}^{t}[f(x, t-s) g(x, s)] d s, \quad t \geq 0 .
$$

*Received May 20, 1965; revised manuscript received August 30, 1965.

${ }^{* *}$ The generalization to the quasi-static theory of linear viscoelastic materials has already been carried out by Gurtin [2]. 
We will have occasion to use Titchmarsh's Theorem:

$$
f * g \equiv 0 \Rightarrow \text { either } f=0 \text { or } g=0 \text { for } t \geq 0 .
$$

For convenience and clarity in presentation, all smoothness and regularity hypotheses on functions and regions are omitted.

We need the following four lemmas, which are trivial extensions of these given in [1]. These lemmas play a role analogous to that of the fundamental lemma of the calculus of variations.

Lемма 2.1 Let $v$ be a sufficiently smooth function on $R \times[0, \infty)$ and suppose

$$
\int_{R}[v * \omega](x, t) d x=0, \quad t \geq 0,
$$

for every arbitrarily smooth function $\omega$ on $R^{c} \times[0, \infty)$ which, together with all its space derivatives, vanishes on $\partial R \times[0, \infty)$ and on $R$ at $t=0$. Then

$$
v=0 \text { on } R^{c} \times[0, \infty) \text {. }
$$

LEмMa 2.2. Let $v$ be sufficiently smooth on $B_{s}^{c} \times[0, \infty)$ and suppose

$$
\int_{B_{\text {. }}}[v * \omega](x, t) d x=0, \quad t \geq 0,
$$

for every arbitrarily smooth function $\omega$ which vanishes on $B_{u} \times[0, \infty)$ and on $R$ at $t=0$. Then

$$
v=0 \text { on } B_{s}^{c} \times[0, \infty) .
$$

Lemma 2.3. Let $v_{i}$ be sufficiently smooth on $B_{u}^{c} \times[0, \infty)$ and suppose

$$
\int_{B_{u}}\left[v_{i} *\left(\omega_{i j} n_{j}\right)\right](x, t) d x=0, \quad t \geq 0,
$$

for every arbitrarily smooth symmetric tensor-valued function $\omega_{i ;}$ which, together with all its space derivatives, vanishes on $B, \times[0, \infty)$ and on $R$ at $t=0$. Then

$$
v_{i}=0 \text { on } B_{u}^{c} \times[0, \infty) .
$$

Lemma 2.4. Let $v_{i}$ be sufficiently smooth on $B_{s}^{c} \times[0, \infty)$ and suppose

$$
\int_{B_{i}}\left[v_{i} * \omega_{i i, j}\right](x, t) d x=0, \quad t \geq 0,
$$

for every arbitrarily smooth symmetric tensor-valued function $\omega_{i i}$ that vanishes on $R$ at $t=0$. Then

$$
v_{i}=0 \text { on } B_{s}^{c} \times[0, \infty) .
$$

Finally, let $\Omega\{\cdot\}$ denote a linear functional on a subset $K$ of a linear space $L$. Further, let

$$
\begin{gathered}
y, y^{\prime} \varepsilon L, \\
y+\lambda y^{\prime} \varepsilon K \quad \text { for every real number } \lambda,
\end{gathered}
$$

*See, for example, Mikusinski [3] for proofs and additional discussion.

${ }^{* *}$ We write $\int_{R} \cdots d x$ for the integral over the set $R$. Thus $\int_{R} \cdots d x$ is a volume integral and $\int_{\partial R} \cdots$ $d x$ is a surface integral. 
and formally define $\delta_{\boldsymbol{y}}, \Omega\{y\}$ on $K$ by

$$
\delta_{y}, \Omega\{y\}=\left.\frac{d}{d \lambda} \Omega\left\{y+\lambda y^{\prime}\right\}\right|_{\lambda=0} .
$$

We say that the variation $\delta \Omega\{\cdot\}$ is zero at $y$ over $K$, and write

$$
\delta \Omega\{y\}=0 \quad \text { over } K
$$

if, and only if, $\delta_{y}, \Omega\{y\}$ exists and equals zero for all $y^{\prime}$ consistent with (2.11).

3. The initial-history problem with mixed boundary conditions. In this section we will formulate the viscoelastic initial-history problem for a large class of boundary conditions. We will also give two equivalent characterizations for the solutions.

Let $\rho(x), b_{i}(x, t), u_{i}(x, t), \epsilon_{i j}(x, t), \tau_{i j}(x, t)$ denote, respectively, the mass density of the medium, the body force per unit mass, the displacements, the strains, and the stresses, all defined for $(x, t) \varepsilon R^{c} \times(-\infty, \infty)$.

The linearized field equations, for a viscoelastic medium which occupies a region $R^{c}$ in space, consist of: the equations of motion.

$$
\tau_{i j, j}+b_{i}=\rho u_{i}^{(2)}, \quad \tau_{i j}=\tau_{j i} ;
$$

the strain-displacement relations

$$
\epsilon_{i i}=u_{(i, i)}
$$

and the stress-strain relations* given by either

$$
\tau_{i j}(x, t)=G_{i j k l}(0) \epsilon_{k l}(x, t)+\int_{0}^{\infty}\left[G_{i j k l}^{(1)}(s) \epsilon_{k l}(x, t-s)\right] d s,
$$

or, equivalently, ${ }^{* *}$ by

$$
\epsilon_{i j}(x, t)=J_{i j k l}(0) \tau_{k l}(x, t)+\int_{0}^{\infty}\left[J_{i j k l}^{(1)}(s) \tau_{k l}(x, t-s)\right] d s .
$$

We assume that the relaxation functions $G_{i j k l}(t)$ and the creep compliance functions $J_{i j k l}(t)$, both defined on $[0, \infty)$, have the symmetry properties:

$$
G_{i j k l}=G_{i i k l}=G_{k l i j}, \quad J_{i j k l}=J_{i i k l}=J_{k l i j} .
$$

Before formulating the problem, we find it convenient to introduce the concepts of admissible and viscoelastic states.

An admissible state $S=\left\{u_{i}, \epsilon_{i j}, \tau_{i j}\right\}$ is an ordered triplet of functions $u_{i}, \epsilon_{i j}, \tau_{i i}$,

*In order to avoid the use of complicated notation, we will assume that the material is homogeneous; however, all of our results can easily be extended to include the inhomogeneous case.

${ }^{* *}$ We tacitly assume that the stress-strain relations are invertible.

†The assumption $G_{i j k l}(t)=G_{k l i j}(t)$ for every $t \geq 0$ is difficult to justify and its validity has been the subject of considerable inquiry. Coleman [4] has shown that, as a consequence of the second law of thermodynamics, the above relation holds when $t=0$ and $t=\infty$. Gurtin and Herrera [5] also obtain this result, but on the assumption that the work done in every deformation is non-negative. Rogers and Pipkin [6] discuss this problem in relation to Onsager's Principle and the consequences of material symmetry. They show, for example, that a material with "handedness" may not have this property. Of course, if the material is isotropic, then $G_{i j k l}(t)=G_{k l i j}(t)$ for $t \geq 0$ on the basis of symmetry alone.

Suffice it to say that we must have this property if we are to obtain variational principles; however, it is still an open question to some investigators as to whether one is justified in making this assumption. 
defined on $R^{c} \times(-\infty, \infty)$, for which $\epsilon_{i i}=\epsilon_{i i}$ and $\tau_{i j}=\tau_{i i}$. The restriction of $S$ to $R^{c} \times(-\infty, t)$, denoted by $S^{t}=\left\{u_{i}^{t}, \epsilon_{i j}^{t}, \tau_{i j}^{t}\right\}$, is called the history of $S$ up to time $t$. If we define addition of states and multiplication of a state by a scalar $\lambda$ through

$$
S+S^{\prime}=\left\{u_{i}+u_{i}^{\prime}, \epsilon_{i j}+\epsilon_{i j}^{\prime}, \tau_{i j}+\tau_{i i}^{\prime}\right\}, \quad \lambda S=\left\{\lambda u_{i}, \lambda \epsilon_{i j}, \lambda \tau_{i j}\right\},
$$

then the set of admissible states is a linear space.

A viscoelastic state $V=\left\{u_{i}, \epsilon_{i j}, \tau_{i j}\right\}$ is an admissible state for which $u_{i}, \epsilon_{i j}, \tau_{i j}$ satisfy the field equations (3.1), (3.2), and (3.3).

Since a viscoelastic material "remembers" its past history through the mechanism of the constitutive relation (3.3), we must, in order to obtain a well-posed problem, prescribe the displacements, strains, and stresses in the body up to some initial time $t_{0}$. Thus our initial data consists of three functions $u_{i}^{\prime \prime}, \epsilon_{i j}^{\prime \prime}, \tau_{i j}^{\prime \prime}$ on $R^{c} \times\left(-\infty, t_{0}\right)$ which, of course, satisfy the field equations. We take $t_{0}=0$, without loss of generality, since the field equations are invariant with respect to translations of the time axis. Finally, we write $V^{\prime \prime}=\left\{u_{i}^{\prime \prime}, \epsilon_{i j}^{\prime}, \tau_{i j}^{\prime \prime}\right\}$ and call $V^{\prime \prime}$ the initial history.

By a solution to the mixed problem, we mean a viscoelastic state $V$ which satisfies the initial history condition

$$
V^{-0}=V^{\prime \prime},
$$

and the boundary conditions

$$
\begin{aligned}
& u_{i}=u_{\imath}^{b} \quad \text { on } B_{u} \times[0, \infty), \\
& T_{i}=\tau_{i j} n_{i}=T_{i}^{b} \text { on } B_{s} \times[0, \infty) \text {, }
\end{aligned}
$$

where $u_{i}^{b}$ and $T_{i}^{b}$ are prescribed functions. If $B_{u}=\partial R\left\{B_{\imath}=\partial R\right\}, V$ is said to be a solution to the displacement $\{$ traction $\}$ problem.

In order to obtain alternate formulations to this problem, we first try to characterize viscoelastic states $V$ which satisfy the initial history condition (3.6), in which case we say that $V$ is a viscoelastic state with the initial history $V^{\prime \prime}$.

Lемма 3.1. $V$ is a viscoelastic state with the initial history $V^{\prime \prime}$ if, and only if, $V$ is admissible and satisfies the initial history condition (3.6), the equations of motion (3.1), the strain-displacement relations (3.2), and either

$$
\tau_{i i}=\tau_{i i}^{+}+\left[G_{i j k l} * \epsilon_{k l}\right]^{(1)}, \quad t \geq 0,
$$

where

$$
\tau_{i i}^{+}(x, t)=\int_{0}^{\infty}\left\{G_{i i k l}^{(1)}(x, t+s) \epsilon_{k l}^{\prime \prime}(x,-s)\right\} d s, \quad t \geq 0,
$$

or, equivalently,

$$
\epsilon_{i j}=\epsilon_{i j}^{+}+\left[J_{i j k l} * \tau_{k l}\right]^{(1)}, \quad t \geq 0
$$

where

$$
\epsilon_{i j}^{+}(x, t)=\int_{0}^{\infty}\left\{J_{i j k l}^{(1)}(x, t+s) \tau_{k i}^{\prime \prime}(x,-s)\right\} d s, \quad t \geq 0 .
$$

${ }^{*}$ Of course, if the initial history vanishes identically the functions $\tau_{i j}^{+}$and $\epsilon_{i j}^{+}$vanish. 
Proof. Suppose $V$ is admissible and satisfies (3.1), (3.2), (3.6), and (3.9a and b). Then, by virtue of the definitions of initial history and viscoelastic state, the "if" part of the assertion is true if we can show that (3.9a and b) implies (3.3a) for $t \geq 0$. This follows at once from (2.1) and a simple change in variable. Hence, $V$ is a viscoelastic state with the initial history $V$ ". To show that the "only if" part of the assertion is true, assume that $V$ is a viscoelastic state with the initial history $V^{\prime \prime}$. Clearly, $V$ is admissible and (3.1), (3.2), and (3.6) hold. Thus we are done if we can show that (3.3a) implies $(3.9 \mathrm{a}$ and $\mathrm{b})$ for $t \geq 0$. We can see that this is so by splitting the range of integration in (3.3a) into the two sets $[0, t]$ and $(t, \infty)$ and using (2.1) and (3.6), together with a simple change of variable, to obtain ( $3.9 \mathrm{a}$ and $\mathrm{b})$. In a similar fashion we can deal with the inverse stress-strain relations. This completes the proof.

Although all smoothness hypotheses have been omitted, we make the following exception: If $S=\left\{u_{i}, \epsilon_{i j}, \tau_{i j}\right\}$ is an admissible state which satisfies the initial history condition (3.6), then we assume that $u_{i}$ is twice differentiable with respect to time* and hence automatically satisfies the initial condition

$$
\begin{aligned}
u_{i}(x, 0) & =\lim _{t \rightarrow 0^{-}} u_{i}^{\prime \prime}(x, t) \stackrel{\text { def }}{=} d_{i}(x), \\
u_{i}^{(1)}(x, 0) & =\lim _{t \rightarrow 0^{-}} u_{i}^{\prime \prime(1)}(x, t) \stackrel{\text { def }}{=} v_{i}(x) .
\end{aligned}
$$

Notice that $d_{i}$ and $v_{i}$, defined through (3.10), (3.11), depend only upon the initial history $V^{\prime \prime}$.

Henceforth, we will denote by $l$ and $g$ the functions on $[0, \infty)$ defined by

$$
l(t) \equiv 1, \quad g(t) \equiv l * l(t)=t, \quad t \geq 0 .
$$

Of course, for any differentiable function $h(t)$ such that $h(0)=0$

$$
l * h=g * h^{(1)} \text {. }
$$

Further let $f_{i}$ be the vector valued function defined on $R^{c} \times[0, \infty)$ by

$$
f_{i}(x, t)=g * b_{i}(x, t)+\rho\left[t v_{i}(x)+d_{i}(x)\right], \quad t \geq 0 .
$$

We now state, without proof, a result due to Ignaczak [7]

LemMa 3.2. Let $\tau_{i j}=\tau_{j i}$. Then $\tau_{i j}$ and $u_{i}$ satisfy the equations of motion (3.1) and the initial condition (3.10), (3.11), for $t \geq 0$ if, and only if,

$$
g * \tau_{i, i}+f_{i}=\rho u_{i}, \quad t \geq 0 .
$$

Lemmas 3.1 and 3.2 enable us to give an alternate formulation of the mixed problem.

Theorem 3.1. Let $S=\left\{u_{i}, \epsilon_{i j}, \tau_{i j}\right\}$ be an admissible state. Then $S$ is a solution to the mixed problem if, and only if: $S$ satisfies the initial history condition (3.6); the equations (3.2), (3.9), and (3.14); and the boundary conditions (3.7).

Proof. Suppose $S$ satisfies (3.2), (3.6), (3.9), (3.14), and (3.7). Then, by Lemmas 3.1 and 3.2, $S$ satisfies the field equations for $t \geq 0$ and the initial history condition (3.6). Thus $S$ is a viscoelastic state with the initial history $V^{\prime \prime}$. But $S$ also satisfies the boundary

*This assumption is quite reasonable since we are seeking displacements $u_{i}$ which satisfy the equations of motion (3.1). Of course, if (3.6) holds then (3.1) is satisfied for $t<0$. 
conditions (3.7); hence, $S$ is a solution to the mixed problem and the "if" part of the theorem is proved. Conversely, to show that the "only if" part is true, assume that $S$ is a solution to the mixed problem. Then, clearly, $S$ is admissible and, moreover, (3.1), (3.2), (3.3), (3.6), and (3.7) hold. Furthermore Lemmas 3.1 and 3.2 imply that (3.9) and (3.14) are satisfied. This completes the proof.

We now characterize solutions to the mixed problem in terms of the initial history $V^{\prime \prime}$ and the stresses $\tau_{i j}$. We say that $\tau_{i j}$ is a stress field corresponding to a solution of the mixed problem if, and only if, $\tau_{i i}=\tau_{i i}$ and $\tau_{i j}$ has the property that there exist functions $u_{i}$ and $\epsilon_{i j}$ such that $\left\{u_{i}, \epsilon_{i j}, \tau_{i j}\right\}$ is a solution to the mixed problem.

Theonem 3.2. Let $\tau_{i j}=\tau_{i i}$. Then $\tau_{i j}$ is a stress field corresponding to a solution of the mixed problem if, and only if, $\tau_{i j}$ satisfies:

$$
\tau_{i i}^{0}=\tau_{i j}^{\prime \prime} ;
$$

and,

$$
\begin{gathered}
g * \tau_{(i k, k i)}-\rho\left[J_{i j k l} * \tau_{k l}\right]^{(1)}=\rho \epsilon_{i i}^{+}-f_{(i, i)}, \quad t \geq 0, \\
g * \tau_{i k, k}=\rho u_{i}^{b}-f_{i}, \text { on } B_{u} \times[0, \infty), \\
T_{i}=\tau_{i j} n_{i}=T_{i}^{b}, \text { on } B_{0} \times[0, \infty)
\end{gathered}
$$

Proof. Suppose $\tau_{i j}$ satisfies (3.15), (3.16), (3.17), and (3.18). Define $u_{i}$ and $\epsilon_{i j}$ as follows: for $t<0$,

$$
u_{i}^{0}=u_{i}^{\prime \prime}, \quad \epsilon_{i i}^{0}=\epsilon_{i j}^{\prime \prime} ;
$$

while for $t \geq 0, u_{i}$ and $\epsilon_{i j}$ are defined, respectively, through (3.14) and (3.9c and d). Then, by (3.15) and (3.19), $\left\{u_{i}, \epsilon_{i i}, \tau_{i j}\right\}$ satisfies (3.6). Moreover, (3.14), (3.16), (3.9c and d), together with (3.6), imply (3.2). Finally, by (3.14), (3.17), and (3.18), the boundary conditions (3.7) are satisfied. Thus, by Theorem 3.1, the "if" part of the theorem is proved. To establish the converse assertion, assume that $\tau_{i i}$ is a stress field corresponding to a solution of the mixed problem. Then by definition there exist functions $u_{i}$ and $\epsilon_{i j}$ such that $\left\{u_{i}, \epsilon_{i j}, \tau_{i j}\right\}$ is a solution to the mixed problem. We now observe that (3.2), (3.9c and d) and (3.14) imply (3.16); (3.14) and (3.7a) imply (3.17); and (3.6) implies (3.15). Moreover, the relations (3.7b) and (3.18) are the same. Thus, by Theorem 3.1, the proof is complete.

Notice that this result depends upon the second of the equivalent relations (3.3a) and $(3.3 \mathrm{~b})$; however, $(3.3 \mathrm{a})$ is satisfied whenever $(3.3 \mathrm{~b})$ is, and vice versa.

4. Variational principles for the mixed problem. The underlying linear space for the two variational principles established in this section is the set of all admissible states.

Theorem 4.1. Let $K$ be the set of all admissible states $S$ that satisfy the initial history condition (3.6). Further, for each $t \geq 0$, define the functional $\Lambda_{t}\{\cdot\}$ on $K$ through

$$
\begin{aligned}
\Lambda_{t}\{S\}= & \frac{1}{2} \int_{R}\left[l * G_{i j k l} * \epsilon_{i j} * \epsilon_{k l}\right](x, t) d x+\frac{1}{2} \int_{R}\left[\rho u_{i} * u_{i}\right](x, t) d x \\
& -\int_{R}\left[g *\left(\tau_{i j}-\tau_{i j}^{+}\right) * \epsilon_{i j}\right](x, t) d x-\int_{R}\left[\left(g * \tau_{i j, j}+f_{i}\right) * u_{i}\right](x, t) d x
\end{aligned}
$$




$$
+\int_{B_{u}}\left[g * T_{i} * u_{i}^{b}\right](x, t) d x+\int_{B_{i}}\left[g *\left(T_{i}-T_{i}^{b}\right) * u_{i}\right](x, t) d x
$$

Then

$$
\delta \Lambda_{t}\{S\}=0 \text { over } K \text { for each } t \geq 0
$$

if, and only if, $S$ is a solution to the mixed problem.

Proof. Let $S^{\prime}=\left\{u_{i}^{\prime}, \epsilon_{i j}^{\prime}, \tau_{i i}^{\prime}\right\}$ be chosen so that $S+\lambda S^{\prime} \varepsilon K$ for all real $\lambda$. Then (4.1), together with (3.4), (3.12), (2.1), (2.12), the divergence theorem, and the properties of the convolution, implies

$$
\begin{aligned}
\delta_{S^{\prime}} \Lambda_{t}\{S\}= & \int_{R}\left[g *\left(u_{(i, j)}-\epsilon_{i j}\right) * \tau_{i j}^{\prime}\right](x, t) d x \\
& -\int_{R}\left[g *\left(\tau_{i j}-\tau_{i j}^{+}-\left[G_{i j k l} * \epsilon_{k l}\right]^{(1)}\right) * \epsilon_{i j}^{\prime}\right](x, t) d x \\
& -\int_{R}\left[\left(g * \tau_{i j, i}+f_{i}-\rho u_{i}\right) * u_{i}^{\prime}\right](x, t) d x \\
& +\int_{B_{u}}\left[g *\left(u_{i}^{b}-u_{i}\right) * T_{i}^{\prime}\right](x, t) d x \\
& +\int_{B_{i}}\left[g *\left(T_{i}-T_{i}^{b}\right) * u_{i}^{\prime}\right](x, t) d x, \quad t \geq 0 .
\end{aligned}
$$

Suppose that $S$ is a solution to the mixed problem. Then, by Theorem 3.1, (4.3), and the choice of $S^{\prime},(4.2)$ holds and the "if" part of the theorem is proved. Conversely, to show that the "only if" part is true, assume that (4.2) holds. Then

$$
\delta_{S}, \Lambda_{t}\{S\}=0 \text { for each } t \geq 0
$$

and for all $S^{\prime}$ such that $S+\lambda S^{\prime} \varepsilon K$ for all real $\lambda$. Clearly, $S^{\prime 0}=\{0,0,0\}$; hence, we can choose $S^{\prime}=\left\{u_{i}^{\prime}, 0,0\right\}$, where $u_{i}^{\prime 0} \equiv 0$ and $u_{i}^{\prime}$, together with all its space derivatives, vanishes on $\partial R \times[0, \infty)$ and on $R$ at $t=0$. Then (4.3) and (4.4) imply

$$
\int_{R}\left[\left(g * \tau_{i j, j}+f_{i}-\rho u_{i}\right) * u_{i}^{\prime}\right](x, t) d x=0, \quad t \geq 0 ;
$$

hence, Lemma 2.1 implies that (3.14) holds. Consider the same choice of $S^{\prime}$ but require now that $u_{i}^{\prime}$ vanish on $B_{u} \times[0, \infty)$. Then (4.3), (4.4), (3.14), Lemma 2.2, (2.2), and (3.12) imply (3.7b). Next choose $S^{\prime}=\left\{0, \epsilon_{i j}^{\prime}, 0\right\}$, where $\epsilon_{i i}^{\prime 0} \equiv 0$ and $\epsilon_{i i}$, together with all its space derivatives, vanishes on $\partial R \times[0, \infty)$ and on $R$ at $t=0$. Clearly, $\epsilon_{i j}^{\prime}$ is symmetric; thus, (4.3), (4.4), Lemma 2.1, (2.2), and (3.12) imply (3.9a). Finally, let $S^{\prime}=\left\{0,0, \tau_{i j}^{\prime}\right\}$, where $\tau_{i j}^{\prime 0} \equiv 0$, and $\tau_{i j}^{\prime}$, together with all its space derivatives, vanishes on $\partial R \times[0, \infty)$ and on $R$ at $t=0$. Then $\tau_{i j}^{\prime}$ is symmetric and (4.3), (4.4), Lemma 2.1, (3.12), and (3.6) imply (3.2). If we now require that $\tau_{i j}^{\prime}$, together with all its space derivatives, vanish on $B, \times[0, \infty)$, we obtain, from, (3.2), (4.3), (4.4), (3.12), (2.2),

*Expanded, the first term on the right hand side of (4.1) has the form

$$
\frac{1}{2} \int_{R} \int_{0}^{t} \int_{0}^{s} \int_{0}^{r}\left\{G_{i i k l}(s-r) \epsilon_{i j}(r-q) \epsilon_{k l}(q)\right\} d q d r d s d x
$$


and Lemma 2.3, the boundary condition (3.7a). Now since (3.2), (3.6), (3.7), (3.9), and (3.14) hold, we appeal to Theorem 3.1 and the proof is complete.

Theorem 4.2. Let $K$ be the set of all admissible states $S$ which satisfy the straindisplacement relations (3.2) and the initial history condition (3.6). Further, for each $t \geq 0$, define the functional $\Theta_{t}\{\cdot\}$ on $K$ through

$$
\begin{aligned}
\Theta_{b}\{S\}= & \int_{R}\left[g * \tau_{i j} *\left(\epsilon_{i i}-\epsilon_{i j}^{+}\right)\right](x, t) d x-\frac{1}{2} \int_{R}\left[l * J_{i j k l} * \tau_{i j} * \tau_{k l}\right](x, t) d x \\
& +\frac{1}{2} \int_{R}\left[\rho u_{i} * u_{i}\right](x, t) d x-\int_{R}\left[f_{i} * u_{i}\right](x, t) d x \\
& -\int_{B_{u}}\left[g * T_{i} *\left(u_{i}-u_{i}^{b}\right)\right](x, t) d x-\int_{B_{\iota}}\left[g * T_{i}^{b} * u_{i}\right](x, t) d x
\end{aligned}
$$

Then

$$
\delta \Theta_{\imath}\{S\}=0 \text { over } K \text { for each } t \geq 0
$$

if, and only if, $S$ is a solution to the mixed problem.

Proof. Let $S^{\prime}=\left\{u_{i}^{\prime}, \epsilon_{i j}^{\prime}, \tau_{i j}^{\prime}\right\}$ be chosen so that $S+\lambda S^{\prime} \varepsilon K$ for all real $\lambda$. Then (4.6), together with (3.2), (3.4), (3.12), (2.1), (2.12), the divergence theorem, and the properties of the convolution, implies

$$
\begin{aligned}
\delta_{S}, \Theta_{\tau}\{S\}= & \int_{R}\left[g *\left(\epsilon_{i i}-\epsilon_{i i}^{+}-\left(J_{i, k l} * \tau_{k l}\right)^{(1)}\right) * \tau_{i i}^{\prime}\right](x, t) d x \\
& -\int_{R}\left[\left(g * \tau_{i j . i}+f_{i}-\rho u_{i}\right) * u_{i}^{\prime}\right](x, t) d x \\
& +\int_{B_{u}}\left[g *\left(u_{i}^{b}-u_{i}\right) * T_{i}^{\prime}\right](x, t) d x \\
& +\int_{B_{0}}\left[g *\left(T_{i}-T_{i}^{b}\right) * u_{i}^{\prime}\right](x, t) d x, \quad t \geq 0 .
\end{aligned}
$$

Suppose $S$ is a solution to the mixed problem. Then, by virtue of Theorem 3.1 and (4.8), (4.7) holds and the "if" part of the theorem is proved. To show that the converse assertion is true, assume that (4.7) holds. Thus

$$
\delta_{S}, \Theta_{t}\{S\}=0, \text { for each } t \geq 0
$$

and for all $S^{\prime}$ such that $S+\lambda S^{\prime} \varepsilon K$ for all real $\lambda$. Clearly, $S^{\prime 0}=\{0,0,0\}$; hence, we can choose $S^{\prime}=\left\{u_{i}^{\prime}, 0,0\right\}$, where $u_{i}^{\prime 0} \equiv 0$ and $u_{i}^{\prime}$, together with all its space derivatives, vanishes on $\partial R \times[0, \infty)$ and on $R$ at $t=0$. Then (4.8), (4.9), and Lemma 2.1 imply (3.14). We now require that $u_{i}^{\prime}$ vanish on $B_{u} \times[0, \infty)$. Then (4.8), (4.9), (3.12), (3.14), (2.2), and Lemma 2.2, imply (3.7b). Next let $S^{\prime}=\left\{0,0, \tau_{i j}^{\prime}\right\}$ where $\tau_{i j}^{\prime 0} \equiv 0$ and $\tau_{i j}^{\prime}$, together with all its space derivatives, vanishes on $\partial R \times[0, \infty)$ and on $R$ at $t=0$. It then follows, from (3.5), (4.8), (4.9), (3.12), (2.2), and Lemma 2.1, that (3.9c) holds. Finally, we require that $\tau_{i j}^{\prime}$, together with all its space derivatives, vanish on $B, \times[0, \infty)$. Then (3.9c), (4.8), (4.9), (3.12), (2.2), and Lemma 2.3 imply (3.7a). We can conclude, since $S \varepsilon K$, that (3.6), (3.7), (3.9), (3.14), and (3.2) hold. An appeal to Theorem 3.1 completes the proof. 
The functionals $\Lambda_{t}\{\cdot\}$ and $\Theta_{t}\{\cdot\}$ which appear in Theorems 4.1 and 4.2 of this paper are the generalizations of those which appear in [1]. Indeed, to obtain these formally from their counterparts we simply make the following replacements: for $\Lambda_{t}\{\cdot\}$, let $\tau_{i j} \rightarrow \tau_{i j}-\tau_{i j}^{+}, \hat{T}_{i} \rightarrow T_{i}^{b}-T_{i}^{+}, f_{i} \rightarrow f_{i}+g * \tau_{i j, j}^{+}$, and $c_{i j k l} g *\{\cdot\} \rightarrow G_{i j k l} * l *\{\cdot\} ;$ while for $\Theta_{t}\{\cdot\}$, let $\epsilon_{i j} \rightarrow \epsilon_{i j}-\epsilon_{i j}^{+}$and $\kappa_{i j k l} g *\{\cdot\} \rightarrow J_{i j k l} * l *\{\cdot\}$. Here $c_{i j k l}$ and $\kappa_{i, k l}$ are, respectively, the elastic moduli and the inverse elastic moduli which appear in [1].

5. A variational principle involving only stresses. We say that $\tau=\left\{\tau_{i i}\right\}$ is an admissible stress field if, and only if, $\tau_{i j}=\tau_{i i}$. The class of all admissible stress fields is the underlying linear space for the next theorem.

Theorem 5.1. Let $K$ be the set of all admissible stress fields $\tau$ for which $\tau_{i i}^{0}=\tau_{i i}^{\prime \prime}$. Further, for each $t \geq 0$, define the functional $\Gamma_{t}\{\cdot\}$ on $K$ through

$$
\begin{aligned}
\Gamma_{i}\{\tau\}= & \frac{1}{2} \int_{R}\left[g * \tau_{i m, m}^{*} \tau_{i k, k}\right](x, t) d x+\frac{1}{2} \int_{R}\left[\rho\left(J_{i j k l} * \tau_{i j}^{*} \tau_{k l}\right)^{(1)}\right](x, t) d x \\
& -\int_{R}\left[\left(f_{(i, j)}-\rho \epsilon_{i j}^{+}\right) * \tau_{i j}\right](x, t) d x+\int_{B_{u}}\left[\left(f_{i}-\rho u_{i}^{b}\right) * T_{i}\right](x, t) d x \\
& +\int_{B,}\left[g *\left(T_{i}{ }^{b}-T_{i}\right) * \tau_{i j, i}\right](x, t) d x
\end{aligned}
$$

Then

$$
\delta \Gamma_{\imath}\{\tau\}=0 \text { over } K \text { for each } t \geq 0
$$

if, and only if, $\tau=\left\{\tau_{i j}\right\}$ is a stress field corresponding to a solution of the mixed problem.

Proof. Let $\tau^{\prime}$ be chosen so that $\tau+\lambda \tau^{\prime} \varepsilon K$ for all real $\lambda$. Then (5.1), together with (3.4), (3.12), (2.1), (2.12), the divergence theorem, and the properties of the convolution, implies

$$
\begin{aligned}
\delta_{\tau^{\prime}} \Gamma_{t}\{\tau\}=-\int_{R}\left[\left(g * \tau_{(i m, m i)}\right.\right. & \left.\left.+f_{(i, j)}-\rho \epsilon_{i j}^{+}-\rho\left(J_{i j k l} * \tau_{k l}\right)^{(1)}\right) * \tau_{i j}^{\prime}\right](x, t) d x \\
& +\int_{B_{u}}\left[\left(g * \tau_{i m, m}+f_{i}-\rho u_{i}^{b}\right) * T_{i}^{\prime}\right](x, t) d x \\
& +\int_{B_{a}}\left[g *\left(T_{i}^{b}-T_{i}\right) * \tau_{i j, j}^{\prime}\right](x, t) d x, \quad t \geq 0 .
\end{aligned}
$$

Suppose $\tau_{i j}$ is a stress field corresponding to a solution of the mixed problem. Then, by virtue of Theorem 3.2, (5.3), and the choice of $\tau,(5.2)$ holds and the "if" part of the theorem is true.

To show that the "only if" part is also true, we assume that (5.2) holds. Then

$$
\delta_{\tau}, \Gamma_{t}\{\tau\}=0 \text { for each } t \geq 0
$$

and for all $\tau^{\prime}$ such that $\tau+\lambda \tau^{\prime} \varepsilon K$ for all real $\lambda$. Then (2.2), (3.5), (5.3), (5.4), Lemmas 2.1, 2.3, and 2.4, together with the fact that $\tau \varepsilon K$, imply that (3.15), (3.16), (3.17), and (3.18) hold. We appeal to Theorem 3.2 to complete the proof.

The functional $\Gamma_{t}\{\cdot\}$ in the above theorem is the generalization of that in [1]. In 
fact, if we replace $f_{(i, j)}$ by $f_{(i, j)}-\rho \epsilon_{i j}^{+}$in the third term and the operation $\kappa_{i j k l}\{\cdot\}$ by $\left[J_{i j k l} *\{\cdot\}\right]^{(1)}$ in the second term of $\Gamma_{t}\{\cdot\}$ in [1], we immediately obtain (5.1).

Acknowledgment. The author is greatly indebted to Professor Morton E. Gurtin who provided the motivation of this problem and supervised its completion. The author also acknowledges valuable discussions with A. C. Pipkin.

The investigation was sponsored by the Office of Naval Research under contract Nonr 562(10).

During the time in which the work was carried out the author held a Texaco Fellowship at Brown University.

\section{REFERENCES}

[1] Gurtin, M. E., Variational principles for linear elastodynamics, Arch. Rational Mech. Anal., 16, 1, 34

[2] Gurtin, M. E., Variational principles in the linear theory of viscoelasticity, Arch. Rational Mech. Anal., 13, 3, 179

[3] Mikusinski, J., Operational Calculus, Pergamon Press, New York, 1959

[4] Coleman, B. D., On thermodynamics, strain impulses, and viscoelasticity, Arch. Rational Mech. Anal., $17,3,230$

[5] Gurtin, M. E., and Herrera, I., On dissipation inequalities and linear viscoelasticity, Quart. Appl. Math., 23, 3, 235

[6] Rogers, T. G., and Pipkin, A. C., Asymmetric relaxation and compliance matrices in linear viscoelasticity, Z. Angew. Math. Phys., 14, 4, 334

[7] Ignaczak, J., A completeness problem for stress equations of motion in the linear elasticity theory, Arch. Mech. Stos., 15, 2, 225 